\title{
Optimal Operation of an Urban Microgrid Using Model Predictive Control Considering Power Quality Improvements
}

Filipe Perez ${ }^{1 *}$

https://orcid.org/0000-0002-7857-7470

\section{Henry Leonardo López-Salamanca ${ }^{1}$}

https://orcid.org/0000-0002-9261-0214

Lúcio de Medeiros ${ }^{1}$

https://orcid.org/0000-0001-7075-8966

\author{
Leonardo Fuchs ${ }^{1}$ \\ https://orcid.org/0000-0001-7103-6746
}

\author{
Pedro Henrique Roesler ${ }^{1}$ \\ https://orcid.org/0000-0002-6992-6309
}

André Pedretti ${ }^{2}$

https://orcid.org/0000-0003-1357-4911

\section{Ricardo Schumacher ${ }^{1}$}

https://orcid.org/0000-0003-3842-9067

${ }^{1}$ Lactec Institute, Power Systems Division, Curitiba, Brazil; ${ }^{2}$ COPEL Distribution, Curitiba, Brazil.

Editor-in-Chief: Alexandre Rasi Aoki

Associate Editor: Clodomiro Unsihuay Vila

Received: 2021.03.15; Accepted: 2021.06.23.

*Correspondence: filipe.perez@lactec.org.br; Tel.: +55-41-3361-6134 (F.P.).

\section{HIGHLIGHTS}

- Optimal Microgrid operation for both grid-connected and island mode.

- Detailed modelling of the distribution system and the generation devices.

- Load demand and irradiation forecasting models for Model Predictive Control solution.

- Power quality improvements concerning planning and operation.

Abstract: Microgrids have been widely applied to improve the energy quality parameters of a distribution system locally, in addition to ensuring the operation of the system in an isolated manner. The Model Predictive Control (MPC) is a great solution to guarantee the operation of the system considering forecasting models and also physical restrictions of the system, which ensure the optimal operation of the Microgrid. However, the construction of a control scheme following the objectives established in order to meet the connected and isolated operation of a Microgrid is still a challenge. This paper proposes the development of an MPC control scheme that assures optimal system operation in connected and islanded mode, improving power quality indexes, ensuring network requirements, and extending battery life cycle. The proposed control operation in the connected mode can attend to the needs of the Microgrid, reducing the impacts of peak demand and the intermittent variations in renewable generation, where a linear objective function is developed for this purpose. In the islanded mode, grid requirements are guaranteed through load shedding, considering improvements in continuity indicators. Forecasting models are implemented considering the MPC approach and a detailed network model is developed. Simulation results highlight the effectiveness of the proposed control strategy.

Keywords: Microgrids; MPC control; Power Quality. 


\section{INTRODUCTION}

The growth of renewable energy sources in recent years is due to the incentive to change the energy matrix by clean sources, modernization of the network, and development of alternative generation technologies. The integration of renewable energy sources coordinated with the use of Energy Storage Systems (ESS) to supply power to a local network is the main objective of the Microgrids. The Microgrids allow better integration of renewable sources, as well as adequate management of the storage elements, which brings improvements in the energy quality of the electrical systems [5-7].

In this context, batteries stand out due to their ability to support the network in many cases, such as peak shaving, voltage and frequency regulation, reactive control, etc. However, the life cycle of the batteries can be greatly reduced according to the chosen operation mode. Therefore, it is necessary to analyze the performance of storage resources to optimize their operation. In fact, the advent of ESS has brought some solutions to minimize the impacts caused by intermittent energy sources, mainly solar and wind. Thus, the application of an ESS can bring improvements in energy quality, minimizing variations in voltage and frequency, providing network support, and improving system reliability and resilience [8-11]. The Microgrids allow better integration of renewable sources, supply of local loads regardless of the network, that is, operation in island mode, through the management of ESS. The benefits generated by the use of Microgrids bring greater reliability and resilience to the distribution system with better energy efficiency [12].

There are many challenges that need to be faced in controlling Microgrids. Unlike conventional networks, Microgrids bring the integration of energy sources in the form of distributed generation, which can cause bidirectional flow of power, jeopardizing the protection and control schemes of these systems. In addition, instability problems, due to the interaction of controls and the transitions to different operation modes (connected or isolated). The reduced inertia of the Microgrids due to the composition of loads and generators, based on power electronic equipment - such as power converters used in renewable sources, energy storage, and even electric vehicles -, brings problems in the system's operating margin, reducing the system's robustness in the face disturbances and load changes. The various factors of uncertainty, associated with load demand and especially in renewable energy sources that have intermittent characteristics. Thus, a reliable system operation needs to involve load forecasting and climate forecasting models for renewable generation. Another important factor is the developed electrical models, where several assumptions are made as balanced three-phase systems, inductive lines, and constant loads, which distances traditional models from reality [13].

To address these challenges, control systems must ensure reliable operation of the system. Thus, currents and voltages must be properly controlled to reduce oscillations, the frequency must be kept within the operating margins in both connected and islanded operations. The power balance must be regulated to maintain the balance between demanded load and generated power, smooth variations for the transition from different operating modes, and quick fault detection. In addition, economic dispatch with power-sharing between generations distributed can reduce operating costs and maintain system reliability. The optimization of operating costs can also include the provision of ancillary services together with the optimal management of the power flow of the entire network. Thus, an adequate control strategy guarantees the proper operation for a Microgrid [14,15].

The main challenge in the operation of the Microgrids is to maintain a safe operation of the system, balancing generation and demand, where the management of the system is done through heuristic algorithms and hysteresis control, which have a simple concept and easy implementation. However, in order to perform the system operation in an optimized way, other control techniques are investigated. The MPC solves optimization problems at each sampling time, in order to determine the operational minimum (economic or technical) considering the physical limits and technical restrictions, such as operating margins. Thus, the MPC includes a feedback feature in the optimization for the system to better deal with uncertainties and disturbances, dealing with operational restrictions, such as the limits of storage capacity or the rate of power variation. The MPC can also incorporate generation forecasts and demand and based on the future behavior of the system it is possible to obtain an optimal operation considering economic and technical criteria. What makes the MPC a very relevant control strategy for Microgrids [1-4].

The mathematical formulation used by the MPC allows the inclusion in the same control problem of logical and continuous variables, in order to consider different modes of operation or energy scenarios (excess or deficit in generation can be considered in the same cost function only by associating binary variables) [4].

It is important to emphasize that the open-loop characteristic of an optimization system does not allow to compensate for uncertainties brought by the proposed model and disturbances. On the other hand, the 
MPC closed-loop feature allows corrective actions to be applied, using measurements to update the optimization problem, allowing the system to operate optimally. The hierarchical control structure of Microgrids, usually composed of 3 levels, allows a good separation in the time-scales of the controlled variables, facilitating the application of the MPC.

Considering the Brazilian context, the interest in the application of ESS has grown considerably since the public strategic call of ANEEL in 2016 ("R\&D Call 21"), which had the objective of proposing technical and commercial arrangements for the evaluation and insertion of Energy Storage Systems (ESS) in the Brazilian electric sector, in an integrated and sustainable way. Considering the increasing insertion of renewable sources, it is envisaged the use of large-scale ESS, once proven its technical and economic viability, resulting in impact reductions and obtaining safe and dispatchable way for energy supply. Then, the discussions presented here are part of the results of one of the R\&D projects of Public Call n. 21 (Copel, 2016), focused on the development of a control system for the management of energy storage systems and consumer loads in the context of intelligent Microgrids as support for the integration of distributed generation. A pilot system will be installed in the distributor's concession area, allowing optimal operation considering renewable sources, battery banks, priority and controllable loads, as well as other elements of automation, control, and power electronics.

The development of an optimal control algorithm is provided, through the development of optimization functions in order to minimize the costs of system operation and meet the network requirements. Two distinct algorithms will be implemented, one for operation in the grid-connected mode and the other for the islanded operation mode. In the first case, the objectives of the operation aim to improve the power quality of the network and in island mode, the main objective is to attend the consumer's power demand guaranteeing service quality requirements and considering battery operation costs. The control structure created is based on a predictive control (MPC) scheme containing a receding horizon, where it is always possible to maintain the optimal operation of the system. The control structure seeks to maintain the proper operation considering uncertainties in the forecast of generated and demanded power.

In this context, the paper proposes a MPC algorithm to operate the Microgrid located in the substation of a distribution feeder, based on forecast model for load demand and irradiation profile, considering the gridconnected operation, combining peak-shaving and Photovoltaic (PV) smoothing operation, and the standalone operation mode to assure frequency and voltage stability in the Microgrid. The MPC is built from an optimization function using a Mixed-Integer Linear Programming (MILP) formulation for both operation modes. Comparing with the MPC solutions proposed for Microgrids in $[2-4,13,16]$, the main contribution in this paper is to propose a MPC control that addresses peak-shaving and PV smoothing operation simultaneously when operating in grid-connected mode, whereas it also comprises the modeling of complex penalization policies when product and service power quality requirements are not attended by the utilities. Then simulation results are presented to highlight the effectiveness of the proposed control strategy.

\section{MICROGRID MODEL}

The Microgrid is composed of a PV array of $200 \mathrm{kWp}$, a lithium-ion battery of $250 \mathrm{~kW}$ of nominal power, and $560 \mathrm{kWh}$ of energy capacity. The equipment is connected in the substation of a distribution system in Faxinal do Céu, Paraná, Brazil, where a load group is selected to be supplied in the stand-alone operation mode. The distribution system consists of two distribution feeders, which are Vila Santa Maria and Piquiri. Figure 1 presents the general scheme of the Microgrid.

The detailed model of the distribution system is depicted in Figure 2, where the segments of medium voltage lines and the transformers in the two feeders are presented. The whole system consists of 89 distribution transformers and 510 consumer units.

To develop a detailed model of the distribution network, the data present in the Distributor Geographic Database (BDGD) was used. The BDGD consists of a series of information regarding the analyzed distribution system, parameters, and technical and operational information concerning the equipment present in the distribution system. Therefore, specific parameters of transformers, regulators, capacitors, switches, and consumer units are provided. The distribution system model includes the medium voltage line segments, the distribution transformers and the substation transformer. Regarding distribution line segments, BDGD provides segment length, number of phases, cable geometry, pole structure, insulation, gauge, and cable material. For equipment, such as distribution transformers, the rated power, connection type, and constructive characteristics are provided. 


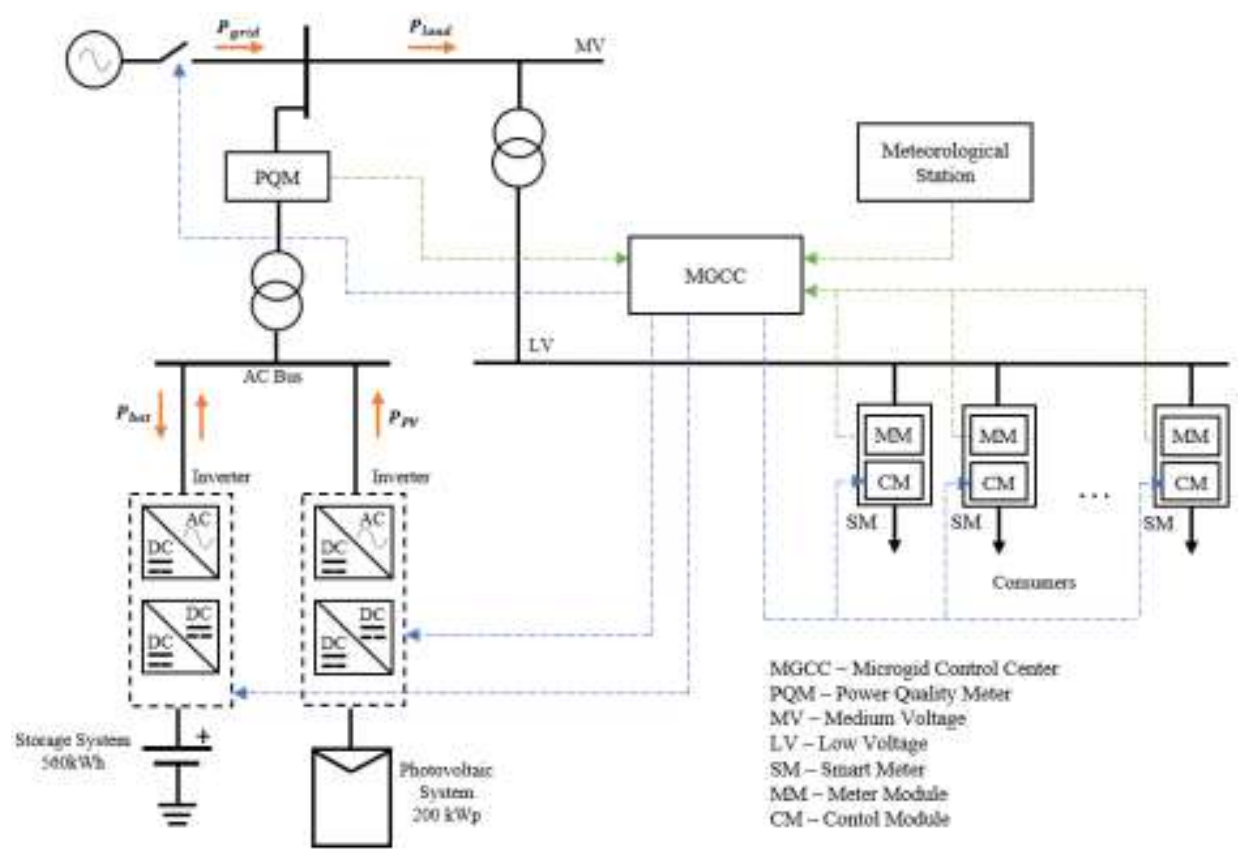

Figure 1. The general scheme of the Microgrid considering the PV array, battery system (ESS), the load group, and the main grid-connection. Also, the communication system of the MGCC.

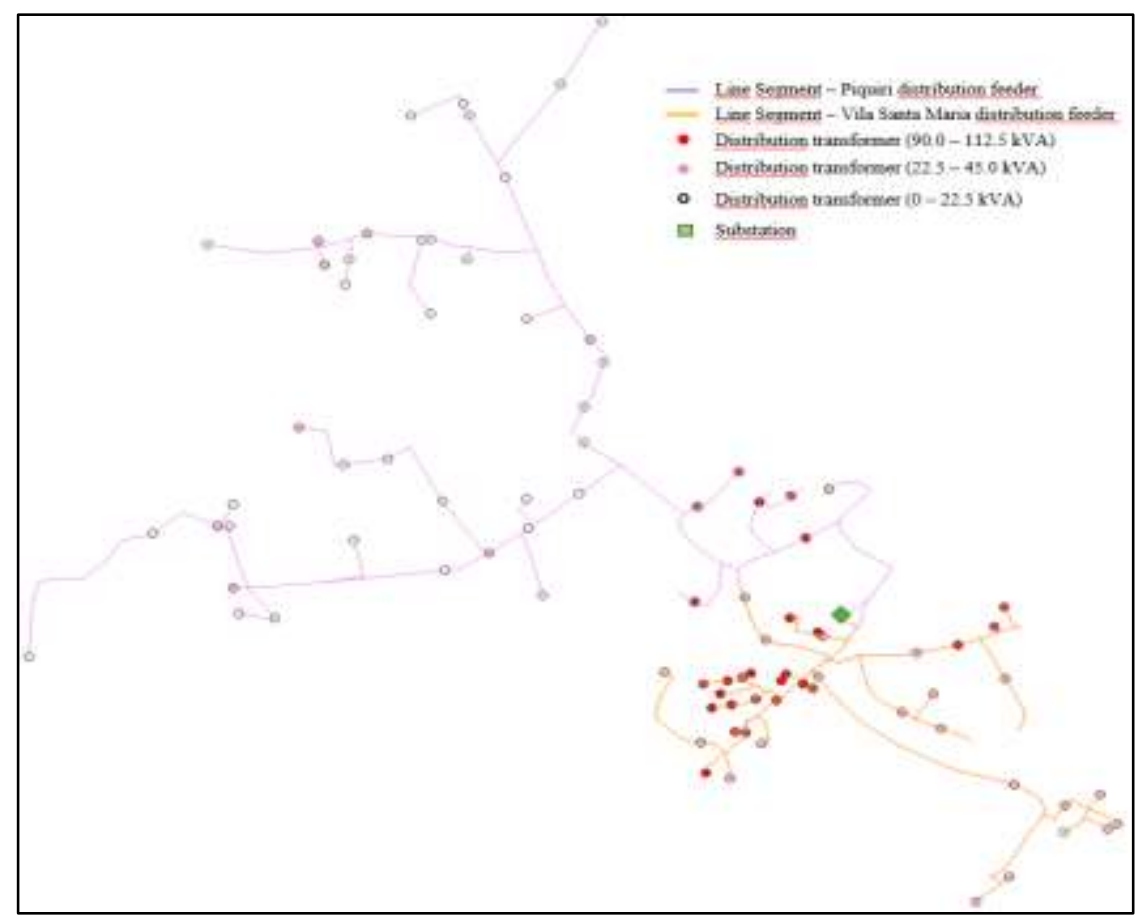

Figure 2. The detailed model of the distribution system, containing the 2 distribution feeders, the cable lines, and the transformers.

The BDGD provides detailed parameters of the equipment and line segments resulting in an improved grid model for power flow software, such as GridLab-D. They are intended for power flow analysis in unbalanced distribution systems, therefore it is possible to model transformers considering the type of connection, the type of installation and electrical parameters, such as rated power, resistance and reactance of the transformer. Thus, the modeling with the BDGD data allows a more detailed and accurate model for electrical studies of the distribution network, making it possible to perform analyses on power flow in each segment or equipment of the system, voltage profile, electrical losses and operational limits of lines or equipment. 


\section{Islanded Microgrid Problem}

In the islanded Microgrid, the system operation provides the balance between generation and consumption. This balance is guaranteed in the proposed controller through switches that disconnect the consumers from the grid and interrupts the PV supply energy. Considering the possibility of not supply energy to consumers, the introduction of a price for load shedding decision is needed. The opposed decision should also be related to price because the ESS is used in these cases. Thus, in the islanded Microgrid operation, two economic aspects are considered for decision making. The first is the cost for the transgression of Brazilian indicators of energy distribution quality service limits, and the second is the ESS operation cost. Both aspects were detailed presented in [17].

The indicators of quality service are presented in the procedures of energy distribution (PRODIST) module 8 [18]. These indicators are utilized to evaluate the performance of utilities that provide energy distribution services in Brazil. Also, these indicators are divided into individual and collective indicators. The individual indicators are applied to indicate whether a consumer must be monetarily compensated for the interruption of energy supply. The collective indicators are calculated based on the individual indicators and are utilized to evaluate the energy distribution utilities. Considering a defined period, the individual indicators are the sum of interruption duration (DIC), the sum of interruption occurrences (FIC), and the longest interruption duration (DMIC). Additionally, defined in a specific period, the collective indicators are the equivalent sum of interruption duration (DEC) and the equivalent sum of interruption occurrences (FEC).

When an individual consumer indicator surpasses the defined limit value the distribution utility must pay a monetary value to the consumer. Therefore, just the greatest compensation is considered. Details about the indicator's value and compensation calculation can be found in [17]. The islanded Microgrid has a PV array as the main generation and the ESS. The PV can be disconnected from the grid through a switch or integrally utilized. The battery responds according to the balance of consumption and generation power. In order to ponder the ESS usage, a price is defined associated with energizing or discharge the battery. In this way, the decision of whether the Microgrid shall supply to the consumers becomes a challenge. Thus, a model utilizing Mixed Integer Linear Problem (MILP) to define the islanded Microgrid operation is proposed. The model function is defining the stated of mentioned switches for each valuated time, as done in [17]. Therefore, this work considers an MPC strategy to minimize the uncertainty of model decisions since these are based on load and PV generation forecasts.

\section{MICROGRID OPERATION AND CONTROL STRATEGY}

In this section, the proposed MPC strategy for optimal Microgrid operation is developed both for gridconnected and island mode. The MPC is built using MILP formulation of optimization function and a forecast model to predict the load demand and the PV generation. The grid-connected operation mode proposes to perform the peak shaving and smooth the PV generation, allowing the optimal battery dispatch considering these objectives and attending to the established restrictions. The islanded operation mode assures the power supply considering load demand and available power in the grid following the grid requirements. The MPC strategy has a forecast horizon of 24 hours ahead with a time step of 10 minutes.

\section{MPC Algorithm for Grid-connected Mode}

Many power companies are employing substantial effort to obtain peak demand reduction with more advanced techniques instead of adopting solely more traditional (and invasive) strategies such as load shedding schemes. One of such promising techniques is peak shaving, whose key idea is described in the following. The power flow balance is crucial to assure proper operation of the Microgrid. The power balance of the proposed Microgrid depicted in Figure 1 in grid-connected mode is given as follows:

$$
P_{\text {grid }}(t)+P_{P V}(t)+P_{\text {bat }}(t)-P_{\text {load }}(t)=0
$$

where $P_{P V}$ is the PV generation, which provides power to the grid, and we consider $P_{P V}(t)>0, P_{\text {load }}$ is the total load demand (including the losses of the system), and only absorbs energy $P_{\text {load }}(t)>0$. The reactive power balance is provided by the power grid. $P_{b a t}$ is the battery power when the battery is providing power $P_{b a t}(t)>0$ (Discharge mode: $P_{b a t}^{d c}$ ), and when it is absorbing power $P_{b a t}(t)<0$ (Charge mode: $\left.P_{b a t}^{c h}\right) . P_{\text {grid }}$ is the power provided by the main grid.

$P_{P V}(t)$ and $P_{\text {load }}(t)$ are the considered perturbations and $P_{b a t}(t)$ is the controlled variable, assumed as the output of the system. Indeed, $P_{\text {load }}(t)$ is intermittent and is given by the power demanded by the group of consumers connected to the system at time instant $t$. The PV generation $P_{P V}(t)$ is also intermittent and is 
determined by Maximum Power Point Tracking (MPPT) of the PV system. $P_{b a t}(t)$ is directly controlled by the power converter of the Microgrid. In this case, the main grid naturally adjusts its power supply $P_{\text {grid }}$ in order to respect the power balance in (1).

The control strategy must consider the physical limitation of the equipment, such as the battery capacity, the overload requirements, perturbations, and power variations. Other aspects, such as frequency and voltage limits and the battery State-Of-Charge (SOC) must also be respected. The MPC algorithm proposed in this section must be capable of providing reference values for battery and main grid operation over a prespecified time horizon, these values are considered to be determined by using measurement and forecasting information of $P_{P V}(t), P_{\text {load }}(t)$, and $S O C(t)$.

Figure 3 depicts a typical demand curve $P_{\text {load }}(t)$ for the group of consumers in Figure 2, and a PV generation curve $P_{P V}(t)$ expected for the $200 \mathrm{kWp}$ PV system, under cloudy weather conditions. It is possible to observe in Figure 3, a significant power peak in the load demand $P_{l o a d}(t)$ around 20 p.m., which may result in higher operational costs, as well as more instability risks.

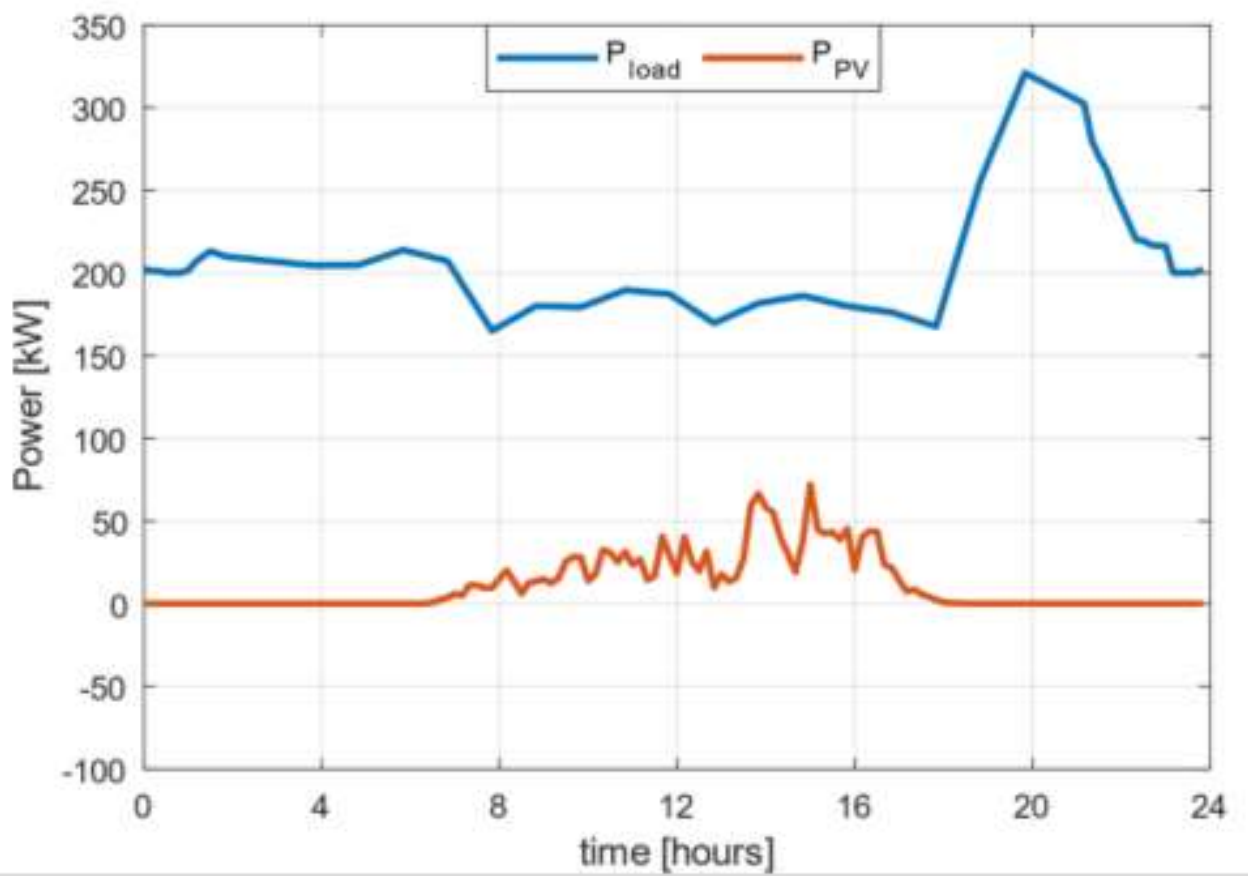

Figure 3. Typical load demand curve and PV generation for a cloudy day.

Around 20 p.m. the PV array is no longer producing energy, however, the ESS may be used to inject a controlled amount of power that provides part of the total demanded power $P_{\text {load }}(t)$. In literature, this is also called "peak-shaving", since the battery can be used to peak shave the demand from the main grid point of view so that $P_{\text {grid }}(t)$ stays within safe limits. With peak demand reduction, reinforcements with generation, distribution, and transmission infrastructure are avoided, especially with the construction of new generation units and with transmission/distribution lines. The avoided reinforcement costs can then be reflected in the price reduction of electricity for all consumers. Indeed, the price reduction is also obtained with effective peak shaving approaches once the necessity of activation of additional generation units during peak consumption moments can be diminished. Such additional generation units are generally far more expensive than the regularly active ones. In addition, they are often based on non-renewable energy sources such as thermal power plants, which are known to be significantly more polluting. In the specific case of Brazil, additional nonrenewable energy sources such as thermal generation are also generally required during peak consumption moments, being such type of generation significantly more polluting and expensive than renewable energy sources such as with hydroelectric power plants. Also, the equipment of the substation is stressed during the peak demand period, and therefore, the power peak reduction can improve the life cycle of the substation equipment.

The intrinsic intermittent nature of the PV generation depicted in Figure 3 highlights the significant power fluctuations, which may bring stability issues for weak grids. Without battery compensation, the main grid has to smooth such fluctuations in order to satisfy the power balance. Nonetheless, we can use the battery to smooth the PV generation, meaning that the combination $P_{P V}(t)+P_{b a t}(t)$ presents a smoother profile, reducing the power grid fluctuations and reducing strong power variations. 
In order to use the battery system to produce both peak shaving as well as smooth PV generation for the proposed Microgrid, without losing hand of tracking total costs involved in using either the main grid and the battery system, we here propose the minimization of the following linear objective function:

$$
\begin{aligned}
J(t)= & \lambda_{\text {energy }} \Delta t \sum_{j=0}^{N_{p}-1} \hat{P}_{\text {grid }}(t+j \mid t)+d \Delta t \sum_{j=0}^{N_{p}-1}\left(P_{\text {bat }}^{d c}(t+j \mid t)-P_{\text {bat }}^{c h}(t+j \mid t)\right) \\
& +\sum_{j=0}^{N_{p}-1} \text { Penalty }(t+j \mid t)+\alpha_{1} \sum_{j=0}^{N_{p}-1}\left|P_{\text {grid }}^{r e f}(t+j \mid t)-\hat{P} P_{\text {grid }}(t+j \mid t)\right| \\
& +\alpha_{2} \sum_{j=1}^{N_{p}}\left|S O C_{\text {ref }}-\operatorname{SOC}(t+j \mid t)\right|
\end{aligned}
$$

where $\lambda_{\text {energy }}$ is the energy cost of the main grid in $\$ / k W h$ ( $\$$ is in the Brazilian currency), $d$ is the battery cost in $\$ / k W h$, and Penalty is a value applied when the system is operating under power peak demand. $P_{\text {grid }}^{\text {ref }}$ is the desired reference given for the main grid and $S O C_{r e f}$ is the reference value for the SOC of the battery, $\alpha_{1}$ and $\alpha_{2}$ are coefficients of the objective function and $\Delta t$ is the time step of the MPC control.

The objective function described above is considered to be minimized for a specific instant $t$, where the key objective is to find the values of $P_{b a t}(t+j \mid t)$ and $\hat{P}_{\text {grid }}(t+j \mid t)$ that produce such minimization. In the MPC formulation, the notation $\hat{P}_{\text {grid }}(t+j \mid t)$ indicates the predicted value of variable $P_{\text {grid }}$ at future instant $t+j$ calculated with the information available at instant $t$.It is important to emphasize that $P_{b a t}(t)$ is not solely determined for time instant $t$ but also over a time horizon determined by $N_{p}=144$ ( $24 \mathrm{~h}$ ahead). $\Delta t=10 \mathrm{~min}$ determines the sampling time between adjacent samples, then the optimization solver has to select the best values for $P_{b a t}(t)$ and $P_{\text {grid }}(t)$ for time instant $t$, but also for the day-ahead interval $N_{p} \Delta t=86400 \mathrm{~s}=24 \mathrm{~h}$, i.e., $P_{\text {bat }}(t+1), P_{\text {bat }}(t+2), \cdots, P_{\text {bat }}(t+143)$ and $\hat{P}_{\text {grid }}(t+1), \hat{P}_{\text {grid }}(t+2), \cdots, \hat{P}_{\text {grid }}(t+143)$. However, since we are proposing to work on a MPC basis, only the actual values obtained for $P_{\text {bat }}(t)$ and $\hat{P}_{\text {grid }}(t)$ are used in the system, and all other 143 values for these variables can be discarded. In the next step, a new set of measurements for $P_{P V}(t), P_{\text {load }}(t)$ and $\operatorname{SOC}(t)$ shall be available, which can then be used within the algorithm to recalculate a new optimal solution for $P_{b a t}(t+j \mid t)$ and $\hat{P}_{\text {grid }}(t+j \mid t), j=0, \cdots, N_{p}-1$. The control scheme recalculates the solution of $J(t)$ each new sampling time instant, based on a new set of available measurements. This control loop is called receding horizon, and improves the system operation, making it more robust against unknown perturbations and forecast errors.

The first term in (1) is related to the operation cost of the main grid according to the power level injected into the grid to supply the load. The second term is used to measure the cost of the battery operation in charge and discharge mode with $P_{b a t}^{c h}(t+j \mid t)<0$ and $P_{b a t}^{d c}(t+j \mid t)>0$. Therefore, $P_{b a t}(t)=P_{b a t}^{c h}(t)+$ $P_{b a t}^{d c}(t)$, where two related binary variables $\delta_{d c}(t)$ and $\delta_{d c}(t)$ are defined in order to determine if the battery is either charging or discharging at time instant $t$. Specifically, we consider $\delta_{d c}(t)=1$ and $\delta_{c h}(t)=0$ if battery is discharging at instant $t$ and $\delta_{d c}(t)=0$ and $\delta_{c h}(t)=1$ if battery is charging. Mathematically, if one assumes that the battery may either provide or absorb a maximum amount of power $P_{b a t}^{\text {max }}$, one can implement such logic by using the following linear inequality constraints for $j=0, \ldots, N_{p}-1$ :

$$
\begin{gathered}
0 \leq P_{b a t}^{d c}(t+j \mid t) \leq P_{b a t}^{\max } \delta_{d c}(t+j \mid t) \\
0 \geq P_{b a t}^{c h}(t+j \mid t) \geq-P_{b a t}^{\max } \delta_{c h}(t+j \mid t) \\
\delta_{c h}(t+j \mid t)+\delta_{\mathrm{dc}}(t+j \mid t) \leq 1
\end{gathered}
$$

The third term in (2) provides the peak shaving operation, proposing an economical penalty penalty $(t+j \mid t)=$ penalty $_{\text {value }}$ in case $\hat{P}_{\text {grid }}(t) \geq P_{\text {grid }}^{\text {lim }}$, and penalty $(t+j \mid t)=0$ otherwise. By defining $\delta_{\text {penalty }}(t)$ as a binary variable that determines the application of this penalty, this logic is embedded to the optimization problem by means of the following linear inequality constraints for $j=0, \ldots, N_{p}-1$ : 


$$
\begin{gathered}
\hat{P}_{\text {grid }}(t+j \mid t)-P_{\text {grid }}^{\text {lim }} \geq L\left(1-\delta_{\text {penalty }}(t+j \mid t)\right) \\
\hat{P}_{\text {grid }}(t+j \mid t)-P_{\text {grid }}^{\text {lim }} \leq(U+\epsilon) \delta_{\text {penalty }}(t+j \mid t)-\epsilon \\
\text { penalty }(t+j \mid t)-\text { penalty }_{\text {value }} \leq U\left(1-\delta_{\text {penalty }}(t+j \mid t)\right) \\
\text { penalty }(t+j \mid t)-\text { penalty }_{\text {value }} \geq L\left(1-\delta_{\text {penalty }}(t+j \mid t)\right) \\
\text { penalty }(t+j \mid t) \leq U\left(\delta_{\text {penalty }}(t+j \mid t)\right) \\
\text { penalty }(t+j \mid t) \geq L\left(\delta_{\text {penalty }}(t+j \mid t)\right)
\end{gathered}
$$

The fourth term in (2) provides the smoothening of the PV generation. In this case, a reference signal $P_{\text {grid }}^{r e f}(t+j \mid t)$ is created for the main grid power $\hat{P}_{\text {grid }}(t+j \mid t)$. Therefore, we define $P_{\text {grid }}^{r e f}(t+j \mid t)=$ movmean $\left(\hat{P}_{\text {load }}(t+j \mid t)-\hat{P}_{P V}(t+j \mid t), k\right)$, where movmean $(X, k)$ is the moving mean operator that returns an array of local $k$-point mean values, where each mean value is calculated over a sliding window of length $k$ across neighboring elements of $X$. Figure 4 shows an example of applying this operator for $\hat{P}_{\text {load }}(t+j \mid t)-$ $\hat{P}_{P V}(t+j \mid t)$ with $k=15$ points. The hats over variables $\hat{P}_{\text {load }}(t+j \mid t)$ and $\hat{P}_{P V}(t+j \mid t)$ emphasize that these two variables are not known beforehand in a real-time application and must therefore be estimated (forecasted) over the horizon $j=1, \ldots, N_{p}$. In order to transform this part into a linear programming expression, we use the following transformation:

$$
\min \left|P_{\text {grid }}^{r e f}(t+j)-\hat{P}_{\text {grid }}(t+j)\right|=\min A+B, \text { given that } A, B \geq 0 \text { and } \hat{P}_{\text {grid }}(t+j)-P_{\text {grid }}^{r e f}(t+j)=A-B
$$

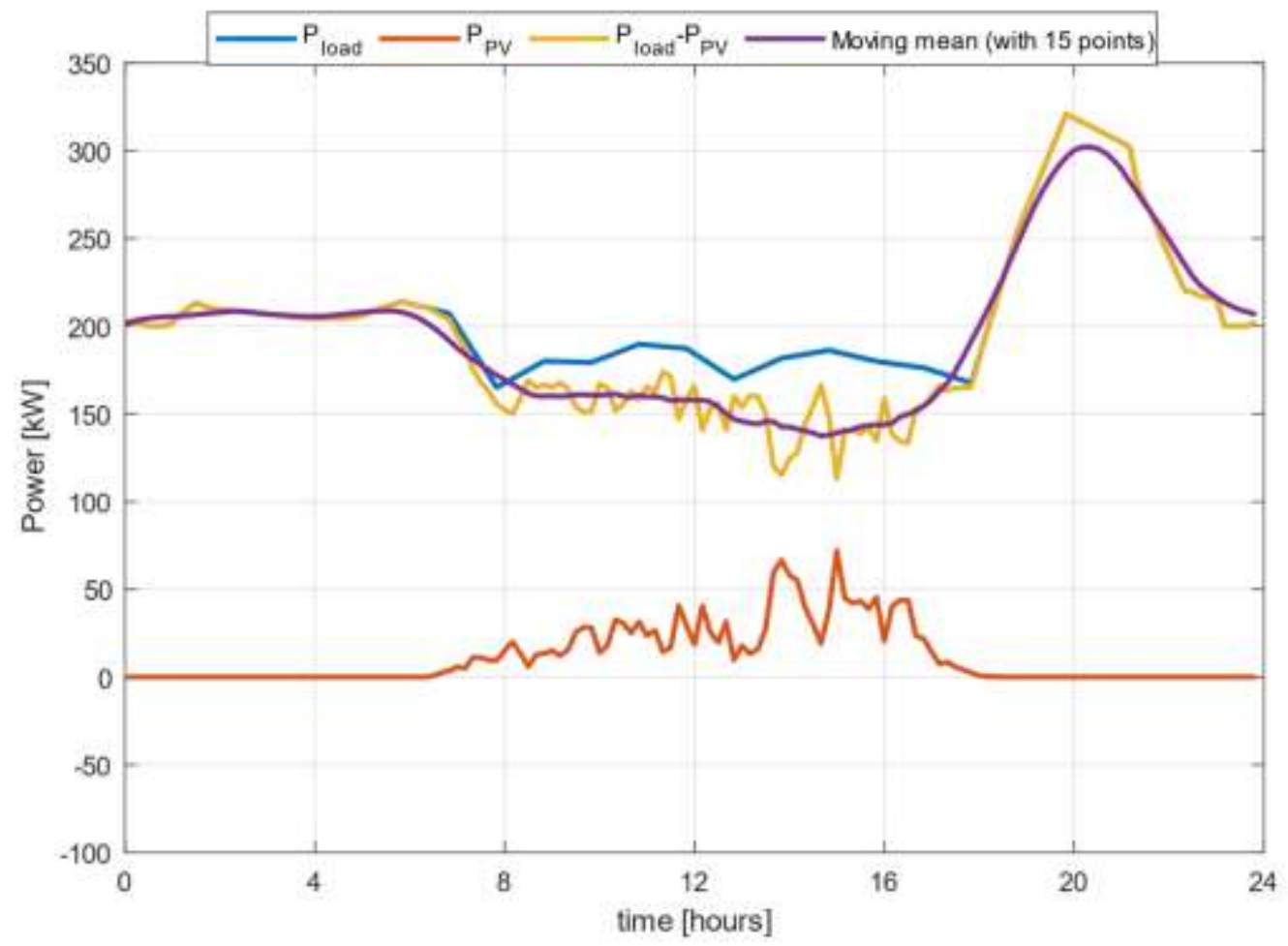

Figure 4. Moving mean example applied to $\hat{P}_{l o a d}(t+j)-\hat{P}_{P V}(t+j)$.

Finally, the fifth term in (2) is applied to maintain the SOC of the battery in the desired operating point, which is the optimal point to allow maximum charge and discharge rate (reference $S O C_{R E F}=50 \%$ ). Therefore, the battery is always ready to operate as required in the control strategy, avoiding the operational limits, such as completely charged or discharged. The SOC estimation can be done through the following equation:

$$
\operatorname{SOC}(t+j+1 \mid t)=\operatorname{SOC}(t+j \mid t)-\Delta t \frac{P_{b a t}^{d c}(t+j \mid t)+P_{b a t}^{c h}(t+j \mid t)}{C_{\max }}
$$

where $C_{\max }$ denotes the maximum battery capacity. In order to protect the battery, we also introduce the SOC limitations as additional linear constraints. 


$$
\mathrm{SOC}_{\min } \leq \operatorname{SOC}(t+j \mid t) \leq \mathrm{SOC}_{\max }
$$

The power balance equation is also included as an important constrain to assure proper operation of the system:

$$
P_{\text {grid }}(t+j \mid t)+P_{\text {bat }}(t+j \mid t)+\hat{P}_{\text {load }}(t+j \mid t)+\hat{P}_{P V}(t+j \mid t)=0, \text { for } j=0, \cdots, N_{p}-1
$$

\section{MPC Algorithm for Islanded Mode}

The proposed MPC solution for controlling islanded Microgrids considers the Model Predictive Control (MPC) approach for solving the control problem and a Mixed Integer Linear Programming (MILP) approach for modeling and solving the optimization problem. In the proposed solution, Microgrid operation is guaranteed by a power balance restriction. Therefore, the predictive output $\hat{y}(t+j \mid t)$ corresponds to predicted battery power, as described by equation (4).

$$
\hat{y}(t+j \mid t)=\frac{1}{\lambda_{D C}(t+j \mid t)}\left\{\frac{1}{\eta_{D C / A C}}\left[\sum_{i=1}^{C} \widehat{D}_{i}(t+j \mid t) u_{i}(t+j \mid t)+\hat{L}(t+j \mid t)\right]-\eta_{P V} \hat{P}_{P V}(t+j \mid t)\right\}
$$

Battery power must be calculated at each instant $(t+j \mid t)$, where $j$ corresponds to the sampling time and $j \in\{1, \ldots, N p\}$, where $N p$ is the prediction horizon. Active power predicted required by the electrical load of each $i$ consumer is represented by variable $\widehat{D}_{i}(t+j \mid t)$ and active power predicted supplied by the PV system, represented by variable $\hat{P}_{P V}(t+j \mid t) . \hat{L}(t+j \mid t)$, corresponds to technical losses predicted. Future control signals $u_{i}(t+j \mid t)$ are described by equation (5). Parameters $\eta_{D C / A C}$ and $\eta_{P V}$ correspond to inverter efficiency and PV system efficiency, respectively. Parameter $\lambda_{D C}$ is relative to electrochemical process efficiency of battery charging $\eta_{c B}$ or battery discharging $\eta_{d B}$ and its predictive value depends on power flow condition through the battery at instant $(t+j \mid t)$. By means of the MILP approach proposed, battery power flow condition is defined by binary variables $\delta_{B S}$ and $\delta_{B C}$ as described by equation (6).

$$
\begin{gathered}
u_{i}(t+j \mid t)=\delta_{\text {open }_{i}}(t+j \mid t) \forall j=1, \ldots, N y \& \forall i=1, \ldots, C \\
\lambda_{D C}(t+j \mid t)=\left\{\begin{array}{c}
\eta_{d B} \forall \delta_{B S}(t+j \mid t)=1 \\
\eta_{C B}{ }^{-1} \forall \delta_{B C}(t+j \mid t)=1
\end{array}\right\}
\end{gathered}
$$

In Equation (5), $C$ is the number of consumers in the Microgrid, and $\delta_{\text {open }_{j}}(t+j \mid t)$ are future binary decision variables. When it is decided to interrupt consumer energy supply $\delta_{\text {open }_{j}}=1$, and when it is decided to keep consumer energy supply $\delta_{\text {open }_{j}}=0$.

Equation (7) describes the objective function formulation for the optimization problem. The goal is to minimize at each iteration $t$ the total cost $Z(t)$ composed by penalty costs of distribution utilities $Z_{\text {int }}(t)$ when power supply requirements are not attended and battery utilization cost, $Z_{\text {bat }}(t)$.

$$
\min (Z(t))=\alpha_{\text {int }} Z_{\text {int }}(t)+\alpha_{\text {bat }} Z_{\text {bat }}(t)
$$

In equation (7), $Z_{\text {int }}(t)$ is calculated as a function of future control signals $u_{i}(t+j \mid t)$ that depends on the connection or disconnection states of the consumer, defined by $\delta_{o p e n_{i}}$, and $Z_{b a t}(t)$ depends on the predictive battery power defined by $\hat{y}(t+j \mid t)$, as previously mentioned. Parameters $\alpha_{i n t}$ and $\alpha_{b a t}$ are coefficients that can be used to prioritize or discard the costs that make up the objective function. In [17], is discussed more details about restrictions formulation that completes the optimization model problem.

To conclude, The MPC control low formulation aims to find the matrix control $U_{j}{ }^{N p}=\left[u_{i}(t+j \mid t) \ldots u_{i}(t+\right.$ $j \mid t)]_{C \times N_{p}}$ that minimizes at each sampling time $t$ the objective function described by equation (7). Applying the receding horizon concept, at each sampling time just the first vector of the optimal control vector $U_{j}^{N p}$ is applied to the system, and the others are discarded. The procedure is repeated at next sampling time knowing new system information. For the proposed solution, this first vector corresponds to connection or disconnection states of consumers at $j+1$. With this MPC approach, it is possible to have an anticipatory action calculated at instant $t$ of what will happen to consumer loads at instant $t+1$. This property is relevant for improve Microgrid operation. 


\section{RESULTS}

\section{Grid-connected Mode}

The MPC algorithm for grid-connected mode operation of the Microgrid is here validated by considering both sunny and cloudy weather conditions. The system described in Figure 1 is built in Matlab/Simulink environment to provide the simulation results. The parameters used for simulation are depicted in Table 1. We also remark that all simulations presented in this section were performed in an Intel(R) Core(TM) i5 2.2 GHz 8 GB RAM laptop.

Table 1. MPC algorithm parameters for grid-connected mode operation.

\begin{tabular}{cl} 
Table 1. MPC algorithm parameters for grid-connected mode operation. & \\
\hline Parameter & Value \\
\hline$\Delta t$ & $600 \mathrm{~s}$ \\
$N_{p}$ & 144 \\
$C_{\max }$ & $560 \mathrm{kWh}$ \\
$S O C(t=0)$ & $50 \%$ \\
$\mathrm{SOC}_{\min }$ & $20 \%$ \\
$\mathrm{SOC}_{\max }$ & $80 \%$ \\
SOC $C_{\text {ref }}$ & $50 \%$ \\
$P_{\text {bat }}$ & $250 \mathrm{~kW}$ \\
$d$ & $0.6269 \$ / \mathrm{kWh}$ \\
$\alpha_{1}$ & $d \Delta t \times 5.1$ \\
$\alpha_{2}$ & $d \Delta t \times 10$ \\
$\lambda_{\text {energy }}$ & $0.0780 \$ / \mathrm{kWh}$ \\
$P_{\text {grid }}^{\text {lim }}$ & $226 \mathrm{~kW}$ \\
penalty & $\$ 500$ \\
$U$ & 3000 \\
$L$ & -3000 \\
$\epsilon$ & 0.01 \\
$k$ & 15 \\
\hline
\end{tabular}

First, we consider a cloudy day. The data set for $P_{\text {load }}(t)$ and $P_{P V}(t)$ is presented in Figure 5 . The other variables are also are also depicted in Figure 5, which correspond to power flows related to $P_{\text {grid }}^{r e f}(t), P_{\text {grid }}(t)$, $P_{b a t}(t)$, and $P_{P V}(t)+P_{b a t}(t)$.

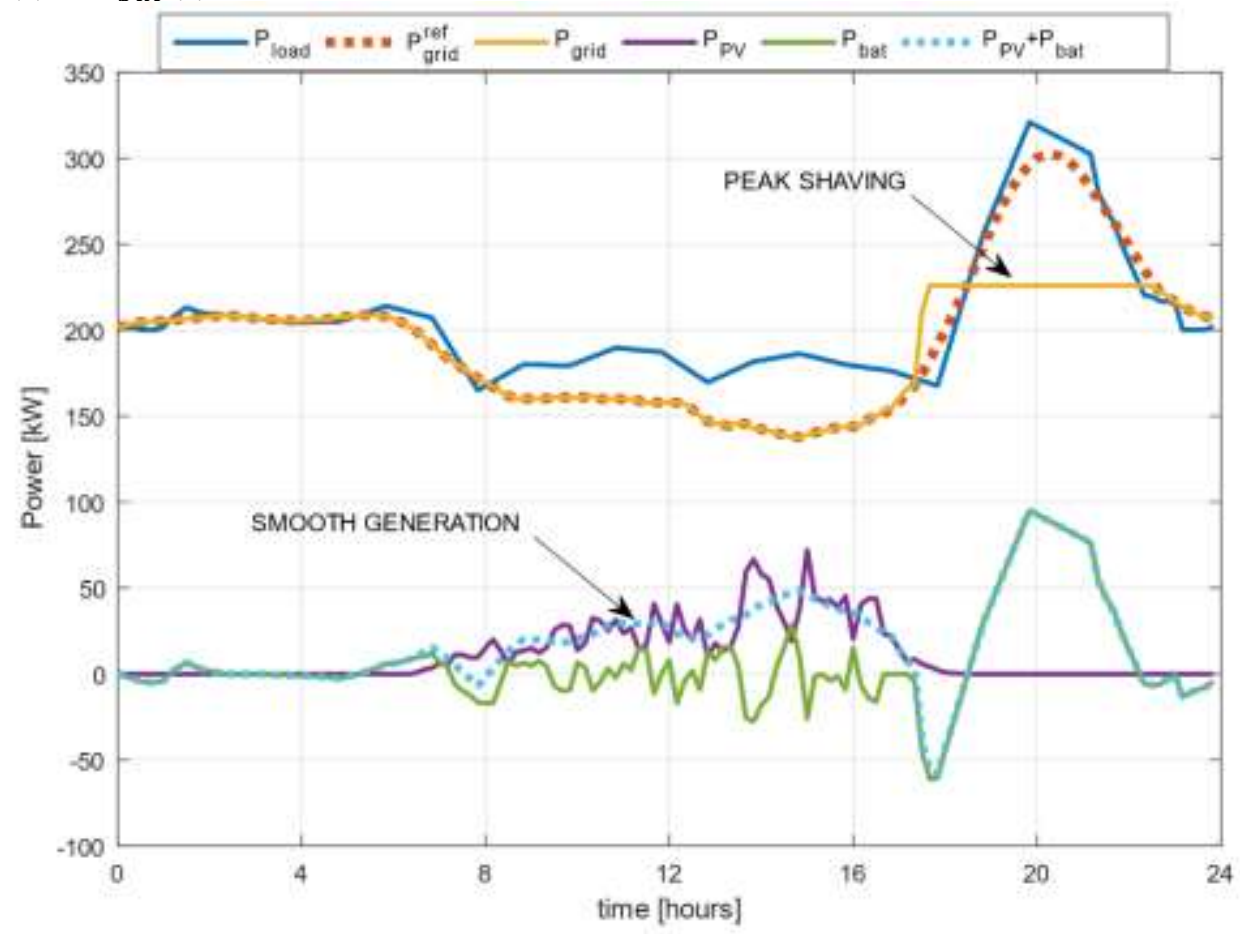

Figure 5. Grid-connected mode: Power flows for a cloudy day. 
The peak shaving is successfully obtained since the battery is consistently activated during the peak demand period in order to maintain $P_{\text {grid }}(t) \leq P_{\text {grid }}^{\text {lim }}=226 \mathrm{~kW}$. Therefore, the penalties have been successfully avoided. On the other hand, the battery has also been used to smooth oscillations from the PV generation, meaning that $P_{\text {grid }}$ will not have to absorb these oscillations. The corresponding SOC evolution from the battery is shown in Figure 6. Before the peak demand moment, it is possible to observe that the battery follows its reference value $S O C_{r e f}=50 \%$. Nonetheless, in order to provide the necessary energy for peak shaving, its minimum allowed value $\mathrm{SOC}_{\min }=20 \%$ has been reached during the peak demand period. Right after, this period the battery starts recharging.

Figure 7 compares the running costs using and not using the Microgrid equipment. First, the total costs are presented when using the Microgrid equipment $(\$ 596.3719)$, which are composed of the costs of extracting power from the grid (\$352.873) - the first term of objective function $J(t)$ in equation (2) - and from using the battery (\$243.4989) - the second term of $J(t)$ in equation (2) -, since penalties have been completely avoided. On the other hand, without using the Microgrid equipment, the main grid becomes solely responsible for feeding the demand, as presented in the second part of Figure 7 . This means that condition $P_{\text {grid }}(t) \leq P_{\text {grid }}^{\text {lim }}=226 \mathrm{~kW}$ will not be satisfied over the entire horizon and penalties during peak moments will occur. This results in the total costs $(\$ 11886.9268)$ of running the system without a Microgrid approximately 20 times greater when compared to the Microgrid installed case.

Next, the simulation is done using data from a sunny day, considering a different irradiation profile. The corresponding results are shown in Figure 8, where peak shaving is once again successfully obtained since the battery is consistently activated during the peak demand period in order to maintain $P_{\text {grid }}(t) \leq P_{\text {grid }}^{\text {lim }}$. The penalties have been successfully avoided just as in the cloudy day case. The battery has also been successfully used to smooth oscillations from the PV generation, although PV generation during sunny days naturally presents a smother shape when compared to its behavior during a cloudy day (compare with Figure 5). Lastly, we also would like to remark that, for both weather scenarios (sunny and cloudy), the proposed MPC implementation took in average less than 3 seconds per iteration to perform all required calculations, which is far less than the utilized $\Delta t=600 \mathrm{~s}$ time-step, assuring the real-time implementation with small computational effort.

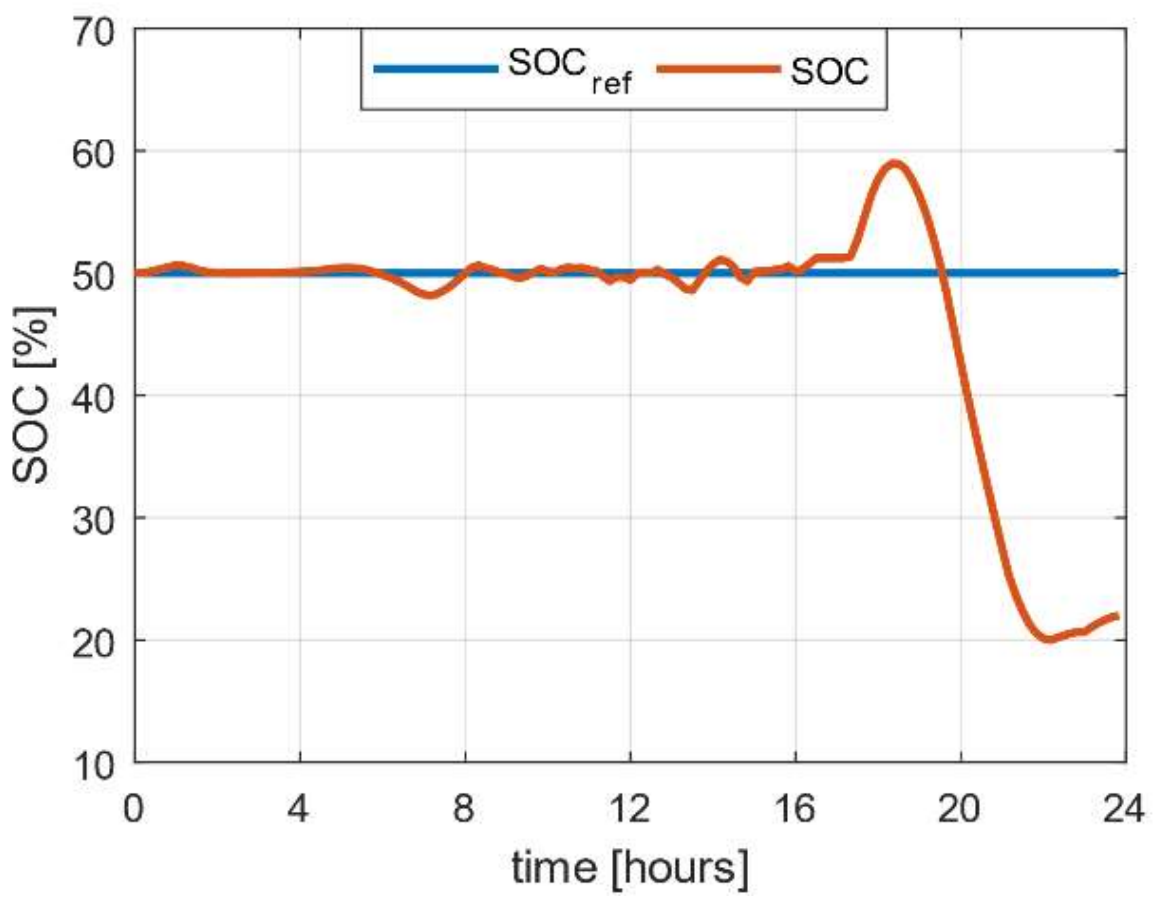

Figure 6. Grid-connected mode: SOC evolution for a cloudy day. 

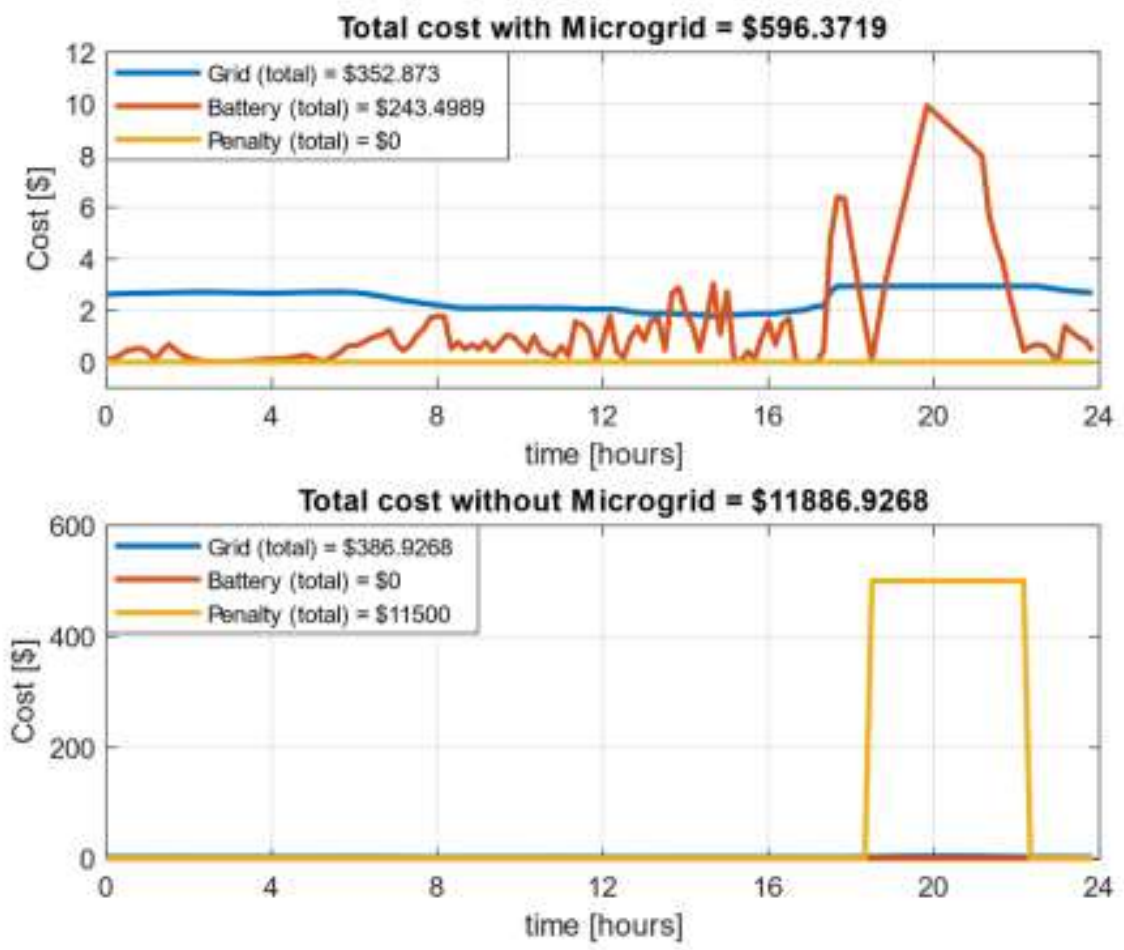

Figure 7. Running costs: Comparison with (top) or without (bottom) installing Microgrid.

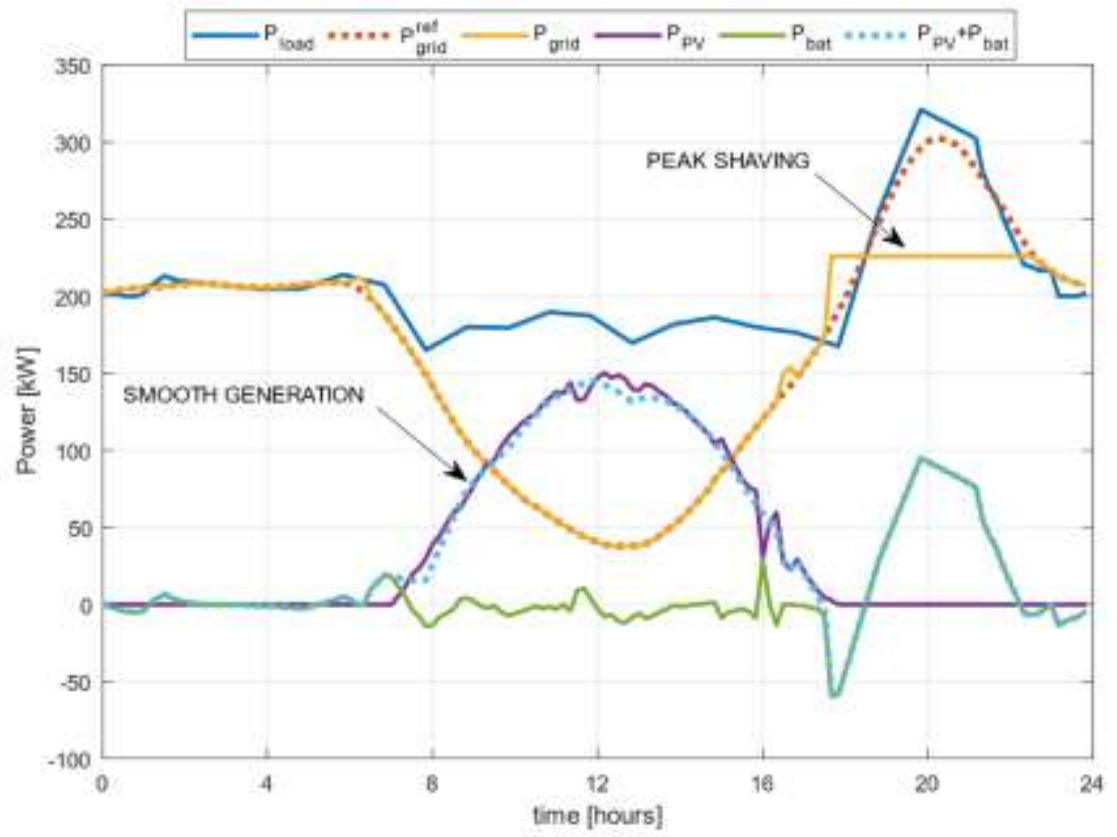

Figure 8. Grid-connected mode: Power flows for a sunny day.

\section{Islanded Mode}

The MPC solution proposed for the control and optimization of an islanded Microgrid is also built in Matlab/Simulink environment. The Simulink provides a notion on how the information will be trade between the Microgrid equipment, also providing an evaluation of ESS electric model response for the decisions of proposed system management. Figure 1 shows the Simulink schematic used in the simulation. 


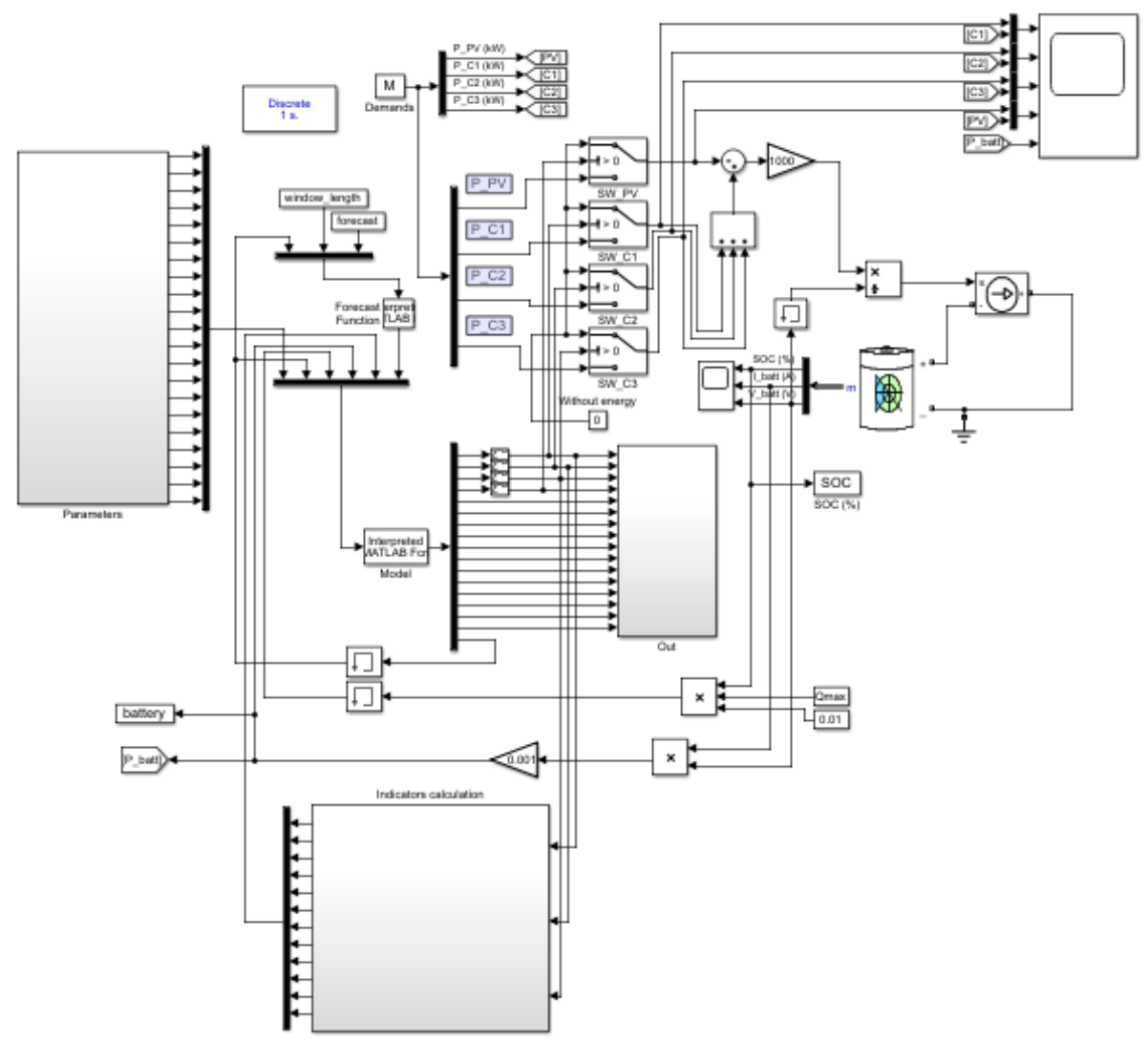

Figure 1. A general overview of Simulink schematic utilized.

It Is important to highlight that the MILP model is solved by the glpk solver using the open-source program called GUSEK. Aiming at the applicability and commercial solution development, the use of an open-source program is an important fact. The function of blocks presented in Figure 9 is utilized to store variables, to update the indicators, to apply the decisions in the switches, to update SOC values and power of SAE, and to provide feedback for the model.

The MPC strategy is applied through the update of model input data for each sampling time. These data are values of generation and demand power of forecast vector, the SOC of ESS, the current power of ESS, and the quality indicators. Table 1 presents the parameter values used in the islanded operation mode.

Table 1. Parameters utilized in island simulation.

\begin{tabular}{ccc}
\hline Parameters & Value & Unit \\
\hline$Q_{\max }, Q_{n}$ & 550 & $\mathrm{kWh}$ \\
$Q_{0}$ & 440 & $\mathrm{kWh}$ \\
$Q_{\min }$ & 110 & $\mathrm{kWh}$ \\
$P_{\max }$ & 250 & $\mathrm{~kW}$ \\
$\eta_{c B}, \eta_{d B}$ & 0.92 & \\
$\eta_{P V}$ & 0.95 & \\
$\eta_{D C / A C}$ & 0.98 & \\
$\alpha_{i n t}, \alpha_{S A E}$ & 1 & Percentual \\
$D o D$ & 80 & \\
$N_{\text {cicles }}$ & 5,000 & $\$$ \\
$C_{S A E}$ & $12,144,000$ & $\$ / \mathrm{kW}$ \\
$T U S D$ & 0.2 & minutes \\
$\Delta t$ & 3 & hours \\
$C$ & 3 & \\
$N y$ & 1 & \\
\hline
\end{tabular}


Table 2 presents the indicators values for each consumer group resulting from the simulation. The cost of indicator compensation is also presented in the table.

Table 2. Values of indicators and indicator compensation for each consumer group.

\begin{tabular}{ccccc} 
Consumer group & DIC (h) & FIC & DMIC (h) & $\mathbf{Z}_{\text {int }}(\mathbf{\$})$ \\
\hline 1 & 1.60 & 4 & 1.30 & 526.66 \\
2 & 0.55 & 5 & 0.30 & 64.51 \\
3 & 0.85 & 9 & 0.15 & 120.86 \\
\hline
\end{tabular}

Table 3 shows the total value of the Microgrid island operation and its components resulting from the simulation. In Table Table $\mathbf{2}$ and Table Table $\mathbf{3} \mathrm{It}$ is observed that the indicators compensation referred to group 1 is higher than the compensation of other groups. This occurred because the indicator compensation is calculated in function of the group average demand. For a better decision evaluation,

Figure 2 illustrates the demand of each group and the power resulting from the decision-making defined by the MPC strategy.

Table 3. Values of Microgrid island operation cost.

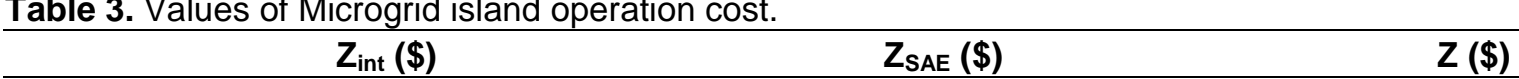

$\begin{array}{lll}702.03 & 195.46 & 897.49\end{array}$

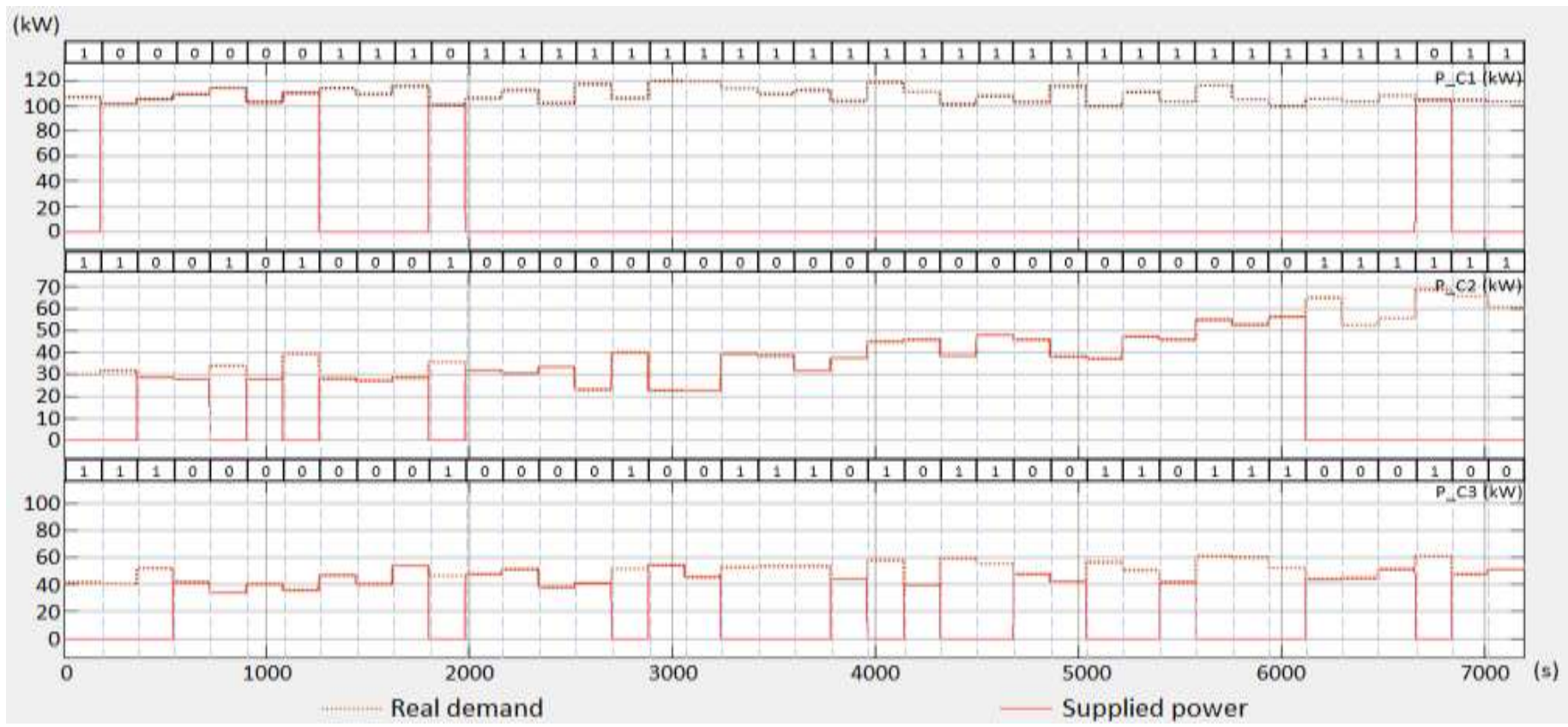

Figure 2. The demand of each consumer and the decision resulting power.

Analyzing

Figure 2, it is possible to conclude that group 1 kept for a longer time without energy supply, even having the most expensive compensation. This result is because the ESS cost to attempt the consumer group is higher than the cost of indicator compensation. Consumer group 3 has a high number of shutdowns in the operation period.

Figure 3 shows the electric parameters of ESS resulting from simulation, such as the SOC, the discharge and charge current, and the ESS voltage. 


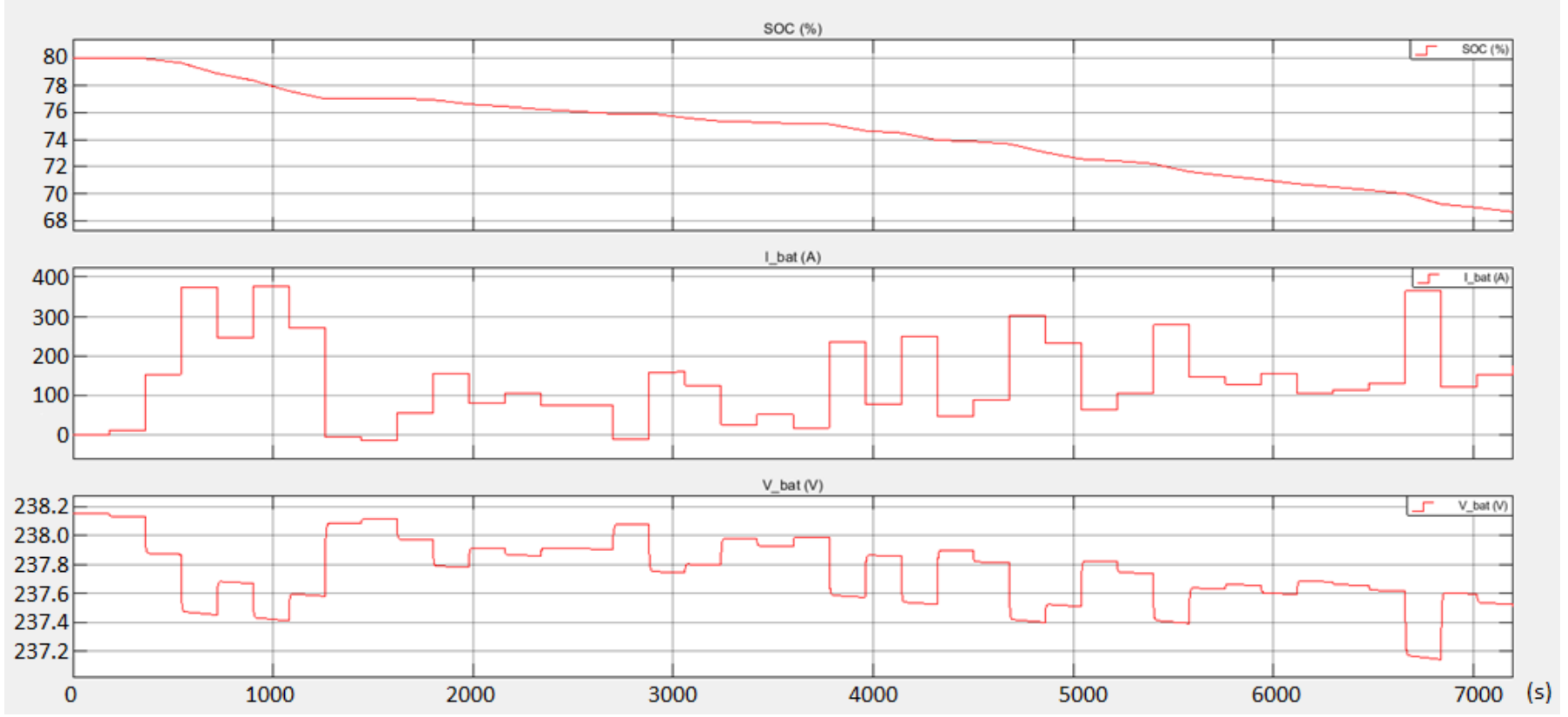

Figure 3. Electrical parameters of ESS resulting from simulation.

In

Figure 3 is possible to observe that the ESS energy is sufficient to meet the energy consumption resulting from the load shedding decisions of control and optimization proposed algorithm. Finally, related to the model convergency time is observed that all times are below 62 seconds. Regarding the frequency of convergency time value, the majority of time values have been between 5 and 25 seconds. In other words, in normal conditions, the model handily meets the limit time requirement of 3 minutes. This limit time value characterizes the energy interrupt time according to PRODIST module n. 8 [18].

\section{CONCLUSION}

This paper proposes a MPC solution for a Microgrid to perform optimal operation both in grid-connected and in islanded mode. The Microgrid is composed of a PV array and a battery storage system (ESS), which inserted in a substation of a distribution system formed by two feeders, Santa Maria and Piquiri, in Faxinal do Céu, a small city in the interior of Paraná Estate. The MPC strategy is structured by a multi-objective function, where the target is to improve power quality by reducing the peak demand impacts and smooth the renewable interment feature in the grid-connected mode. In the islanded mode, the multi-objective function is built to properly attend to the load demand inside of service quality. In both operation modes, the battery costs are considered aiming to save its life cycle, and the restrictions are given by the operating margins and equipment limitation, in addition to the power flow balance.

Simulation results present the control performance of the Microgrid. In the grid-connected mode, the system is able to operate the ESS, such that the objective of peak shaving and renewable generation smoothing is accomplished. Different scenarios are performed to highlight the effectiveness of the proposed MPC strategy comparing the minimal operation costs, even considering the cloudy days. In the Islanded mode, the Microgrid is able to maintain the quality indicators by properly suppling the consumers of the grid as shown in simulation results, which is a promising solution to control and optimize islanded Microgrids in similar regulatory contexts

The MPC control and optimization formulation comprise the modeling of complex penalization policies when product and service power quality requirements are not fully attended by the utilities. Therefore, we may conclude that the proposed MPC solution improve the system operation and assure power quality in grid-connected and standalone operation. Further studies carrying out to improve convergency times will be included in the formulation, delay times associated with smart meters data transmission, and evaluation of voltage profile along with distribution feeders. Complementary, efforts are to be addressed to implement and validate the proposed solution in a Real Time Digital Simulator (RTDS) environment. 
Acknowledgements: This work has been supported by the Companhia Paranaense de Energia - COPEL research and technological development program, through the PD 02866-0442/2016 project, regulated by ANEEL.

\section{REFERENCES}

1. Parisio A, Rikos E, Glielmo L. A model predictive control approach to microgrid operation optimization. IEEE Trans Control Syst Technol. 2014;22(5):1813-27.

2. Del Real AJ, Arce A, Bordons C. Hybrid model predictive control of a two-generator power plant integrating photovoltaic panels and a fuel cell. In: 2007 46th IEEE Conference on Decision and Control. 2007.

3. Arnold M, Negenborn RR, Andersson G, De Schutter B. Model-based predictive control applied to multi-carrier energy systems. In: 2009 IEEE Power \& Energy Society General Meeting. IEEE; 2009. p. 1-8.

4. Bordons C, Garcia-Torres F, Ridao MA. Model Predictive Control of Microgrids. Springer; 2020.

5. Lasseter RH. Smart distribution: Coupled microgrids. Proc IEEE. 2011;99(6):1074-82.

6. Perez F, lovine A, Damm G, Galai-Dol L, Ribeiro PF. Stability Analysis of a DC MicroGrid for a Smart Railway Station Integrating Renewable Sources. IEEE Trans Control Syst Technol. 2019.

7. Zubieta LE. Are microgrids the future of energy?: DC microgrids from concept to demonstration to deployment. IEEE Electrif Mag. 2016;4(2):37-44.

8. Jadeja R, Ved A, Trivedi T, Khanduja G. Control of Power Electronic Converters in AC Microgrid. Power Syst. 2020;27(11):329-55.

9. Dragicevic T, Lu X, Vasquez JC, Guerrero JM. DC Microgrids - Part I: A Review of Control Strategies and Stabilization Techniques. IEEE Trans Power Electron. 2016;31(7):4876-91.

10. Justo JJ, Mwasilu F, Lee J, Jung JW. AC-microgrids versus DC-microgrids with distributed energy resources: A review. Renew Sustain Energy Rev. 2013;24:387-405.

11. Shuai Z, Sun Y, Shen ZJ, Tian W, Tu C, Li Y, et al. Microgrid stability: Classification and a review. Renew Sustain Energy Rev. 2016;58:167-79.

12. Perez F, De Medeiros L, Pedretti A. Active and Reactive Power control in a grid-connected Microgrid with Energy Storage Management. In: 2019 IEEE PES Conference on Innovative Smart Grid Technologies, ISGT Latin America 2019. 2019.

13. Salamanca HLL. Controle e otimização de microrredes em baixa tensão no contexto brasileiro. 2018;

14. Perez F, Damm G, Lamnabhi-Lagarrigue F, Ribeiro P, Monaro R. Adaptive Variable Synthetic Inertia from a Virtual Synchronous Machine Providing Ancillary Services for an AC MicroGrid. In: 21st IFAC world congress. 2020.

15. Perez F. Control of AC/DC Microgrids with Renewables in the Context of Smart Grids: Including Ancillary Services and Electric Mobility. Université Paris-Saclay; Universidade federal de Itajuba. Brésil; 2020.

16. Morstyn T, Hredzak B, Aguilera RP, Agelidis VG. Model predictive control for distributed microgrid battery energy storage systems. IEEE Trans Control Syst Technol. 2017;26(3):1107-14.

17. Roesler PH, López-Salamanca HL, de Medeiros L, Pedretti A, Tortelli OL. Load Management Optimization for Islanded Microgrids under Brazilian Regulatory Normative. In: 2019 IEEE PES Innovative Smart Grid Technologies Conference-Latin America (ISGT Latin America). IEEE; 2019. p. 1-6.

18. ANEEL P de D de E. Elétrica no Sistema Elétrico Nacional-PRODIST: Módulo 8-Qualidade de Energia Elétrica. Revisão. 2007;5:76.

2021 by the authors. Submitted for possible open access publication under the terms and conditions of the Creative Commons Attribution (CC BY NC) license (https://creativecommons.org/licenses/by-nc/4.0/). 